\title{
Editorial: Modularisierung der Bildung: Flexibilität, aber zu welchem Preis?
}

\section{Gianni Ghisla}

1. Die Idee der Modularisierung in der Bildung ist an sich bestechend und «verführerisch». Auf Anhieb verspricht sie, einen zentralen Anspruch moderner Bildung einlösen zu können, nämlich deren effektive Anpassung an die Bedürfnisse sowohl der Individuen auf der einen als auch der unterschiedlichen gesellschaftlichen Instanzen auf der anderen Seite. Offensichtlich eine ideale Perspektive zur Erneuerung der in der westlichen Welt in die Jahre gekommenen Bildungssysteme, die Mühe bekunden, sich auf die gesellschaftlichen Veränderungen einzustellen. In der Tat lassen sich in den letzten dreissig Jahren in der europäischen Schullandschaft vielfältige Bemühungen ausmachen, die Bildung auf modularer Basis neu zu gestalten. Solche Anstrengungen reichen von der Neukonzipierung von einzelnen Bereichen, z.B. in der beruflichen Weiterbildung, über die Umstrukturierung ganzer Bildungssysteme auf nationaler Ebene, so z.B. in Spanien oder Schottland in den 80er und 90er Jahren, bis zur supranationalen Umgestaltung der Hochschulstrukturen mit der Einführung des ECTS-Systems im Rahmen des von der Europäischen Union (EU) gesteuerten Bologna-Prozess.

Auch in der Schweiz ist eine Modularisierungslandschaft im Entstehen, v.a. im Berufs- und Weiterbildungsbereich. Vertraut man auf das Urteil von über 700 Berufsbildungsexperten, welche in einer Delphi-Studie befragt wurden, so soll sich die Tendenz zum modularen Bildungsangebot bis 2020 noch verstärken. Dannzumal wird nämlich das Berufsbildungsystem, vorab auf der tertiären Stufe, nicht nur breiter im inhaltlichen Angebot und problemlösungsorientierter im didaktischen Ansatz, sondern eben auch modularer in der Organisation sein (Bieri, Longchamp et al., 2004, p. 2 u. 24).

Das beinahe magische Begleitwort zu den Modularisierungsbestrebungen heisst «Flexibilität»: Flexible Anpassung und Diversifizierung von Inhalten und Methoden an die sich ständig veränderden Anforderungen der Gesellschaft und der Arbeitswelt, flexible Individualisierung der Bildungsparcours auf der Basis von immer vielfältigeren persönlichen und beruflichen Biographien. Somit liest sich die Tendenz zur Modularisierung von Bildungsprozessen als eine Komponente der Modernisierungsstrategie postmoderner Gesellschaften (Gonon, 
2002), wovon man sich gleichzeitig die Rationalisierung und Optimierung von Schulsystemen und die Einlösung des immer währenden pädagogischen Ideals der Differenzierung verspricht. Dies ist die eine, positive Seite der Medaille. Deren Schattenseite lässt die optimistischen Erwartungen an den modularen Ansatz auf ein notwendig realistisches Niveau sinken, denn bei der Praxisbewährung haben auch die Modularisierungsstrategien nicht nur ihre Schwächen offen gelegt, sondern ebenso ihre ambivalente Natur zum Vorschein gebracht: Wenn sie einerseits durchaus das Potenzial zu einer individuell gerechteren und somit auch humaneren Ausgestaltung der Bildung aufweisen, so können sie auf der anderen Seite neue Formen der Abhängigkeit und der Unsicherheit generieren.

Derlei Probleme und Ambivalenzen werden auch in allen Beiträgen dieses Heftes ersichtlich, so etwa in der Aufarbeitung der konkreten Erfahrungen in Schottland von Matthias Pilz, bei der Diskussion der Modularisierungsstrategie als bildungs- bzw. ordnungspolitisches Instrument der administrativen Steuerung von Bildungssystemen von Dietmar Frommberger, bei der Analyse der durch Modularisierung verursachten Veränderung der klassischen Ausbildnerrolle von Luca Bausch oder schliesslich bei der Diskussion des Beitrags der Informations- und Kommunikationstechnologien (IKT) an die modulare Gestaltung von Ausbildungsgängen von Lorenzo Cantoni und Luca Botturi.

Bei diesen einführenden Gedanken geht es darum, den Rahmen für die kritische Reflexion solcher Probleme abzustecken. Nach einer kurzen Darstellung wichtiger Erfahrungen und Tendenzen aus den letzten Jahrzehnten wird deshalb eine begriffliche Klärung versucht. Die Aufmerksamkeit gilt danach einer knappen gesellschaftlichen Kontextualisierung des Modulansatzes, die die Skizzierung einiger wichtiger Fragestellungen in politischer, epistemologischer und didaktisch-pädagogischer Hinsicht ermöglicht.

2. Die Begriffe «Modul» und «Modularisierung» finden, aus der Technik und Architektur entlehnt, erst seit wenigen Jahrzehnten Eingang in den pädagogischen Diskurs und in die curriculare Organisation von Lehr- und Lernprozessen. Mit den verhaltenstheoretischen Arbeiten von B. F. Skinner begann in den 60er Jahren an amerikanischen Universitäten die Einführung von Modulen im Rahmen von programmierten Unterrichtssequenzen, die von den Prinzipien der Unterteilung von Lerninhalten und Lernprozessen in einzelne überschaubare und kontrollierbare Teile ausgingen (Bünning, Hortsch et al., 2000)1 ${ }^{1}$. Danach war aber die Ausbreitung ziemlich intensiv und das Modulkonzept fand v.a. im Berufsbildungsbereich einen grossen Anklang. Dafür Weg weisend erwies sich die Aktivität der International Labour Organisation (ILO), die weltweit die Idee der modularen Bildung lancierte. So entwickelten sowohl öffentliche Schulsysteme als auch private Anbieter in den meisten europäischen Ländern erste Bildungsgänge v.a. in der Fortbildung und in spezifischen beruflichen Bereichen wo sektorielle oder partielle Qualifikationen gefragt waren. Alsbald begegnet man aber der Diskussion von der Umgestaltung des gesamten Bildungssystems auf modu- 
larer Basis, so auch in der Schweiz, mit ersten Vorschlägen, in den frühen 70er Jahren, zu einem "Gesamtkonzept des schweizerischen Bildungswesens». Als zukunftsträchtige Lösung für die postobligatorische Schule wurde damals ein Modell der lebenslangen, rekurrenten Bildung auf der Basis eines flexiblen Modulsystems grundsätzlich frei zugänglicher und frei kombinierbarer Unterrichtseinheiten in Aussicht gestellt (Gretler, Haag et al., 1972). Was sich in der Schweiz nie konkretisieren sollte, fand andernorts mehr Anklang, sodass sich in den 80er und 90er Jahren in etlichen Ländern, so z.B. in Grossbritannien, Spanien oder Holland, Vorhaben zur radikalen Umgestaltung der Bildungsysteme, nicht nur im Bereich der Berufsbildung, durchsetzten (vgl. dazu Ertl \& Sloane, 2003). Gleichzeitig und unter dem Druck der zunehmenden Mobilität und der Internationalisierung des Bildunsgwesens haben neben der ILO auch andere internationale Organisationen wie die OECD den Modularisierungsdiskurs forciert. In Europa nimmt die Europäische Union (EU) eine Schlüsselrolle ein, indem sie eine koordinierte Umstrukturierung des gesamten tertiären Bildungswesens auf der Basis eines «European Qualification Framework» (EQF) anstrebt. Diese Umgestaltung ist bereits weit fortgeschritten im Hochschulwesen, wo im Rahmen des Bolognaprozesses das «European Credit Transfert System» (ECTS) ${ }^{2}$ eingeführt wird, weitet sich aber neuerdings auch auf den berufsbildenden Sektor aus, wo sich im Rahmen des Kopenhagenerprozesses ein «European Credits for Vocational Education and Training» (ECVET) im Aufbau befindet ${ }^{3}$. Credits im Sinne des ECTS und der ECVET stellen gewissermassen eine europäische "Bildungswährung» dar und sind an sich nicht einfach mit Modulen gleichzusetzen, aber sie setzen praktisch eine Modularisierung des Ausbildungsangebots voraus. Denn die notwendige Transparenz und Vergleichbarkeit der erlangten Qualifikationen als Bedingung für eine breite, international wirksame Anerkennung scheint am besten auf modularer Basis erreichbar zu sein. Die europäische Bildungspolitik zielt demnach nicht primär auf eine Reform der Bildungssysteme, sondern vielmehr auf deren Konvergenz und Kompatibilität. Im Hochschulwesen ist in der Tat die Einführung des ECTS relativ rasch vorangegangen, indessen stösst die Umsetzung des Systems im berufsbildenden Bereich aufgrund der sehr grossen Unterschiede in den einzelnen Ländern auf heiklere Hindernisse. Die Tendenz zu einer systematischen Modularisierung des tertiären Bildungsbereichs scheint indes in der Schweiz wie in Europa unbestritten zu sein.

In der Schweiz fand nach den ersten Diskussionen aus den 70er Jahren das modulare Konzept als globale Reformstrategie kaum mehr Beachtung. Hingegen wurden zahlreiche Versuche, vorab in der beruflichen Fortbildung, lanciert ${ }^{4}$. So entwickelte z.B. Wettstein 1984 im Kanton Zürich ein «Baukastensystem zur beruflichen Weiterbildung für Arbeitslose» (Wettstein, 2005), aber auch in wirtschaftlichen Kreisen fand das Baukastensystem zunehmend Anklang. Auf die potenziellen Möglichkeiten des Modells wurde so auch die Politik aufmerksam. Am 17. Juni 1993 verlangte die Nationalrätin Judith Stamm mit einer Motion 
die Verwirklichung «einer ganzheitlichen und anerkannten Aus-, Fort- und Weiterbildung im Baukastensystem». Wohl nicht zufälligerweise kam die Initiative von Frauenkreisen, wurde doch die Frage der Neuqualifizierung von vielen Frauen zur Eingliederung ins Beschäftigungssystem immer aktueller. Aufgrund von konzeptuellen Vorarbeiten erteilte dann das Bundesamt für Industrie, Gewerbe und Arbeit (BIGA) 1996 der Schweizerischen Gesellschaft für angewandte Berufsbildungsforschung (SGAB) den Auftrag zur Schaffung einer "Schweizerischen Modulzentrale», die unter Leitung des Bundesamtes eine Modulbörse einrichten und sämtliche Fragen der Anerkennung regeln sollte. Die dreijährige Pilotphase wurde mit dem Vorschlag der definitiven Einführung des Konzepts abgeschlossen und zwar sollte die Modulzentrale als Verbundaufgabe mit einer massgebenden finanziellen Beteiligung von Bund und Kantonen verwirklicht werden (AAVV, 1999). Trotz eines weiterhin regen Interesses seitens der Wirtschaft, musste der anfängliche Optimismus aber einer zunehmenden Distanzierung vom Vorhaben weichen. Vorab die öffentliche Hand scheute vor einer zu profilierten und klaren Verantwortungsnahme zurück und reduzierte die Fördermittel. Das Bundesamt für Berufsbildung und Technologie (BBT) ${ }^{5}$ verabschiedete zwar noch im Jahre 2002 «Richtlinien für die modulare Berufsbildung», aber das Projekt einer schweizerischen Modulzentrale gilt vorerst als gescheitert. Zweierlei Gründe scheinen dafür entscheidend zu sein: Zum einen haben sich bedeutende Wirtschaftsverbände (so z.B. der schweizerische Gewerbeverband - SGV) gegen eine staatliche Einflussnahme und für eine Regelung der beruflichen Weiterbildung durch die Berufsverbände und den freien Markt ausgesprochen (Wettstein, 2005) ${ }^{6}$, zum anderen wurden kritische Stimmen bezüglich der Adäquatheit des vorgeschlagenen Konzepts ${ }^{7}$ (Dubs, 1999; Pütz, 1997) und zu dessen verfrühten Einführung laut (Dubs, 1998). Auch der darauf gegründete Verein «modula», dem es gelang bis 2002 eine ansehnliche Datenbank mit 1500 Modulen und 120 Anbietern aufzubauen, konnte offenbar ohne Unterstützung des Bundes nicht überleben ${ }^{8}$.

Mit der Zurücknahme einer aktiven Rolle seitens des Staates in der Regelung und Koordination ist zwar die einheitliche Basis für eine branchenüberschreitende Beanspruchung von modularen Angeboten in der beruflichen Weiterbildung nicht zustande gekommen, dennoch scheint sich das Prinzip der Modularisierung im Weiterbildungsbereich weitgehend durchzusetzen. Allerdings sind somit auch Tür und Tor für die Anwendung von unzähligen Definitionen, Konzepten und Modellen der Modularisierung geöffnet worden, sodass ein Überblick über die zahlreichen konkreten Angebote und Erfahrungen zunehmend schwieriger wird. Jenseits des Weiterbildungsbereichs sind aber auch konkrete Versuche auszumachen, so in der beruflichen Grundbildung für die Ausbildung in Informatik ${ }^{9}$ oder auch in anderen Berufen, welche zur Zeit die Programme ihrer Ausbildung revidieren ${ }^{10}$. Es ist anzunehmen, dass auch die Bestrebungen auf europäischer Ebene, ein Kreditsystem für die Berufsbildung (ECVET) einzuführen, neben der Ausbildung im Tertiären, auch die berufliche Grundbil- 
dung im Sekundarbereich mitprägen wird. Noch sehr zaghaft sind hingegen die Modularisierungsversuche im obligatorischen Schulbereich ${ }^{11}$.

3. Modulare Modelle und konkrete Erfahrungen bei deren Umsetzung lassen sich nicht einfach darstellen, was nicht zuletzt auf eine uneinheitliche Verwendung des Modulbegriffs zurückzuführen ist. Deshalb scheint ein Klärungsversuch durchaus sinnvoll zu sein ${ }^{12}$. Im englischen Sprachraum wird zumeist folgende einfachste Definition verwendet: «Modules are self-contained units, which in an educational context, provide students with specific learning experiences.» (Badley \& Marshall, 1995, p. 15) Damit werden Module sehr offen definiert als inhaltlich abgegrenzte Einheiten, die Lernerfahrungen ermöglichen. Ein solches Verständnis von Modul unterscheidet sich aber in keiner Weise vom Begriff didaktische Einheit, der im pädagogischen Diskurs europäischer Prägung seit je breite Verwendung geniesst. Eine terminologische Präzisierung mit zusätzlichen Kriterien drängt sich deshalb auf. So gilt es zuerst ein Modul als Teil eines Ganzen anzusehen, also als Teileinheit, die nur mit Bezug zum Ganzen Sinn erlangt. Dieser ganzheitliche Bezug sollte im Voraus, also bei der Konstruktion eines Moduls, gesichert sein, so z.B. in Form eines Berufsprofils, eines Ausbildungsprofils oder einer Gesamtqualifikation. In einer extrem offenen Modulkonzeption (vgl. weiter unten) ist es aber denkbar, dass dieser Bezug individuell hergestellt wird, d.h. etwa, dass Module auf dem Bildungsmarkt einfach zugänglich sind und es dem individuellen Nachfrager anheim gestellt bleibt, sie zu einem «personalisierten Puzzle» zusammenzustellen. Zweitens spielt das Kriterium der Beurteilung eine wichtige Rolle: Letzten Endes unterscheidet sich ein Modul von einer traditionellen Lern- oder didaktischen Einheit dadurch, dass die Leistung nach dessen Abschluss geprüft und zertifiziert wird und entsprechend von unterschiedlichen Institutionen anerkannt werden kann.

Folgende Aspekte sollten im Weiteren sinnvollerweise zur Abgrenzung des Begriffs beigezogen werden ${ }^{13}$ :

- Verwertbarkeit: Ist ein Modul als solches oder nur im Rahmen einer Gesamtausbildung verwertbar? Besteht also die Möglichkeit, es in einem weiteren Ausbildungsrahmen anerkennen zu lassen und so zu kumulieren (im Sinne der «unité capitalisable» bzw. der "credit accumulation»)?

- Dimension: In der Regel wird ein Modul nicht nur inhaltlich sondern auch nach dem Arbeits- oder Lernaufwand (workload) definiert. So kann die durchschnittliche Dauer zwischen 40 und 500 Stunden variieren, wobei sich in Europa eine Dauer zwischen 40 und 80 Stunden als Regel festgesetzt hat 14.

- Standardisierung: Um Anerkennung zu ermöglichen muss Transparenz gewährleistet werden, v.a. der Beurteilungskriterien, da die einfache Absolvierung eines Moduls für dessen Zertifizierung nicht ausreichen kann. D.h., ein Modul muss anhand von Kriterien bzw. Indikatoren an Standards gebunden $\operatorname{sein}^{15}$. 
- Kompetenzorientierung: In der Regel wird mit einem Modul nicht einfach ein bestimmtes (deklaratives)Wissen vermittelt und zertifiziert, sondern es kommen Fähigkeiten und Haltungen hinzu. Wie Kompetenzen näher definiert werden, hängt jeweils stark vom zu Grunde liegenden Kompetenzbegriff ab.

Zentral für die Diskussion von modularen Modellen ist eine Typologisierung aus dem Blickwinkel der jeweiligen bildungspolitischen Strategie, die zur Veränderung von Bildungssystemen oder Teilen davon angestrebt wird. So schlägt Deissinger drei strategische Perspektiven vor, die, etwas abgewandelt, auch im Beitrag von Matthias Pilz in diesem Heft übernommen werden (Deissinger, 1996) ${ }^{16}$ : Die erste betrifft die Absicht, bestehende Bildungsprofile mit Modulen zu bestimmten Inhalten und Kompetenzen zu ergänzen bzw. auszuweiten. Dementsprechend spricht Deissinger vom Expansionskonzept. Die zweite zielt auf die Möglichkeit ab, bestehende Ausbildungsprofile neu zu konzipieren: so z.B. Ausund Fortbildung oder Allgemein- und Berufsbildung zu verschränken oder neue Berufsprofile zu generieren. Allerdings müssen die Module in ein vorgegebenes Profil oder in eine vorgegebene Gesamtqualifikation integriert sein und können nur in diesem Rahmen am Bildungs- und Arbeitsmarkt verwertet werden. Hiezu wird der Begriff Differenzierungskonzept ${ }^{17}$ verwendet. Die dritte Variante wird als Fragmentierungskonzept bezeichnet. Sie geht davon aus, dass Module als in sich eigenständige Einheiten konzipiert werden, die nicht im Voraus zu einem bestimmten Profil gehören und somit einzeln am Bildungs- und Arbeitsmarkt verwertet werden können. Die sinnvolle Kombination der Module wird den einzelnen Subjekten überlassen. Dies ist die extremste Variante der Modularisierung, deren Anwendung an sich die vollständige Neustrukturierung bestehender, von vorbestimmten Berufsprofilen oder Gesamtqualifikationen ausgehenden Bildungssysteme zur Folge hat. Die Modularisierungstendenzen in verschiedenen europäischen Ländern wurden ausgehend von dieser strategischen Kategorisierung von verschiedenen Autoren vergleichend untersucht. Ertl u. Sloane haben etwa Spanien, Frankreich, Schottland und Holland analysiert (Ertl \& Sloane, 2003), Pilz vertieft die Situation in Schottland und Deutschland in diesem Heft $^{18}$. Nachdem man etwa in Schottland und Spanien eine grundlegende Umstrukturierung des Bildungssystems nach einem radikalen modularen Ansatz in die Wege geleitet hat, ist man zusehends davon abgekommen. Das Fragmentierungsmodell scheint dementsprechend an seiner Praxistauglichkeit zu scheitern, da die grenzenlose Möglichkeit der Modulkombination nicht nur Unüberschaubarkeit, sondern auch Schwierigkeiten im Nachweis von spezifischen Kompetenzen und nicht zuletzt eine Überforderung der einzelnen Akteure zur Folge hat. Demgegenüber wird zunehmend versucht, anhand von Moduldesigns im Sinne des Differenzierungskonzepts verschiedene Branchen oder Sektoren des Bildungswesens neu zu konzipieren oder miteinander zu verschränken. So z.B. wird die Weiterbildung immer häufiger modular aufgebaut, wobei damit auch 
die Überwindung der Trennung zwischen Aus- und Fortbildung angestrebt wird. In ähnlicher Absicht lassen sich Versuche zur Verzahnung von Allgemein- und beruflicher Bildung beobachten.

Die Befunde aus diesen Untersuchungen erlauben es, einige Trends in der Entwicklung von modularen Reformkonzepten auf europäischer Ebene hervorzuheben.

- Deutlich zu erkennen ist eine Modularisierung in der beruflichen, etwa berufsbegleitenden Weiterbildung, generell in der Erwachsenenbildung und teilweise in der beruflichen Grundbildung ${ }^{19}$. Dabei sind bedeutsame Impulse zur Schaffung eines europäischen Berufsbildungsraumes von der Einführung von ECVET seitens der EU zu erwarten.

- Ebenfalls weit fortgeschritten ist Modularisierung in der tertiären Bildung, insbesondere im Bereich der Hochschulen, der Fachhochschulen und der höheren Fachausbildung. Wiederum entscheidend ist dabei das ECTS, das von der EU gefördert wird.

- Modularisierung geht weitgehend mit Standardisierung 20 einher, d.h. modulare Systeme begünstigen die Standardisierung bzw. die inhaltliche und formale Regelung von Ausbildungsgängen und somit auch jene Ansätze zur Qualitätssicherung, die eben möglichst breit anerkannte und überprüfbare Standards voraussetzen.

- Nach ersten Versuchen kommt der Einsatz radikaler Modularisierungsmodelle als Strategie zur Umstrukturierung und Modernisierung der Bildung kaum mehr in Frage. Vielmehr kommen flexiblere Mischformen zum Zuge, in denen differenzierte Zugänge je nach Reformzielsetzung bzw. nach Bildungssektor angepasst werden können.

Diese Tendenzen zur Modularisierung bilden eine Komponente eines generelleren Trends zur nachfrage- und outputorientierten Gestaltung von Bildungssystemen, denn, wie von Frommberger in seinem Beitrag ausführlich dargelegt, liegt es sozusagen im Wesen von modularen Ansätzen nachfrageorientiert zu sein.

4. Die dargelegten Tendenzen zur Modernisierung der europäischen Bildungssysteme auf modularer Basis müssen vor dem Hintergrund der gesellschaftlichen Entwicklungen der letzten Jahrzehnte betrachtet werden ${ }^{21}$. Mit der in diesem Beitrag gebotenen Knappheit lassen sich einige wichtige und prägende Aspekte dieser Entwicklung mit den potentiellen Eigenarten modularer Modelle direkt in Beziehung setzen ${ }^{22}$ :

- Lebensläufe werden zunehmend entstandardisiert und individualisiert. Damit verbunden ist die fortschreitende Vielfalt und Heterogenität sozialer und kultureller Lebenswelten, die kaum mehr kontinuierlich verlaufen ${ }^{23}$. Entsprechend hoch sind die Erwartungen an die Bildungssysteme, die vermehrt individuellen Ansprüchen gerecht werden und zugleich auf unvorhersehbare Lebenssituationen und berufliche Anforderungen vorbereiten müssen. 
- Viele der traditionellen Berufsprofile haben keinen Bestand mehr, sodass sich auch die Berufskarrieren kaum mehr nach klassischen Mustern mit einem Lebenslauf decken. Die technologische Entwicklung einerseits und die zunehmende, durch die Internationalisierung und Unstabilität der Märkte verursachte Unsicherheit andererseits, haben innerhalb weniger Jahre die traditionelle Berufsstruktur und die damit verbundene Qualifikationsstruktur radikal verändert. Damit einher geht der Druck zu einer ständigen und lebenslangen Anpassung der für die produktiven Tätigkeiten notwendigen Kompetenzen.

- Das Wissen ist offensichtlich auf dem Wege zum wichtigsten Faktor sämtlicher gesellschaftlicher Systeme zu werden. Sowohl eine kontinuierliche $\mathrm{Zu}$ nahme und Neubestimmung der Wissensbestände als auch die potentielle, weltweite Verfügbarkeit des Wissens sind prägende Merkmale der Wissensgesellschaft, die die Bildungssysteme vor leicht einsehbare Herausforderungen stellen.

- Besonderer Aufmerksamkeit bedürfen die Kommunikationstechnologien, welche einerseits das traditionelle Verständnis und den symbolischen Wert von Raum und Zeit in Frage stellen und andererseits gerade auch den Bildungssystemen vielversprechende Ressourcen, etwa im Bereich von interaktiven Lernszenarien, zur Verfügung stellen.

All diese Aspekte, die den Wandel unserer Epoche wesentlich mitprägen, haben es in sich, traditionelle Sicherheiten aufzubrechen und die Menschen vor neue Herausforderungen zu stellen, die allesamt mit einer erhöhten Anpassungsfähigkeit zu tun haben. Im Folgenden wird versucht, synthetisch die potentiellen Antworten modularer Bildungssysteme auf diese Herausforderungen aufzulisten. Gleichzeitig werden Probleme und Risiken aufgezeigt ${ }^{24}$.

\begin{tabular}{|c|c|c|}
\hline $\begin{array}{l}\text { Aspekte und Herausforderungen } \\
\text { des epochalen Wandels }\end{array}$ & $\begin{array}{l}\text { Mögliche Antworten modularer } \\
\text { Systeme (Vorteile und Chancen) }\end{array}$ & Probleme und Risiken \\
\hline $\begin{array}{l}\text { Individualisierung der } \\
\text { Lebensläufe, } \\
\text { Entstandardisierung der } \\
\text { Lebenswelten } \\
\text { Destrukturierung der } \\
\text { Berufsprofile } \\
\text { Entwicklung und } \\
\text { Verfügbarkeit des Wissens } \\
\text { Kommunikations- } \\
\text { technologien }\end{array}$ & $\begin{array}{l}\text { Extreme Flexibilität: Anpassung } \\
\text { an die Bedürfnisse und Bildungs- } \\
\text { voraussetzungen der Lernenden, } \\
\text { an die Anforderungen der kollek- } \\
\text { tiven Akteuren und an die tech- } \\
\text { nische/wirtschaftliche Entwick- } \\
\text { lung } \\
\text { Begünstigung von Mobilität: } \\
\text { durch Lernortunabhängigkeit, } \\
\text { Vergleichbarkeit und erleichterte } \\
\text { Anerkennung und Verwertung } \\
\text { der formal, non-formal und } \\
\text { informal erworbenen Qualifika- } \\
\text { tionen }\end{array}$ & $\begin{array}{l}\text { Fragmentierung } \\
\text { der Bildungsinhalte: Verlust von } \\
\text { Kohärenz und Sinn, Bildungs- } \\
\text { taylorismus, Qualifikations- } \\
\text { sammelsurium } \\
\text { Funktionalisierung } \\
\text { der Bildungsinhalte und der } \\
\text { Bildungsprozesse } \\
\begin{array}{l}\text { Normierung, Homogenisierung } \\
\text { der Bildungsinhalte }\end{array}\end{array}$ \\
\hline
\end{tabular}




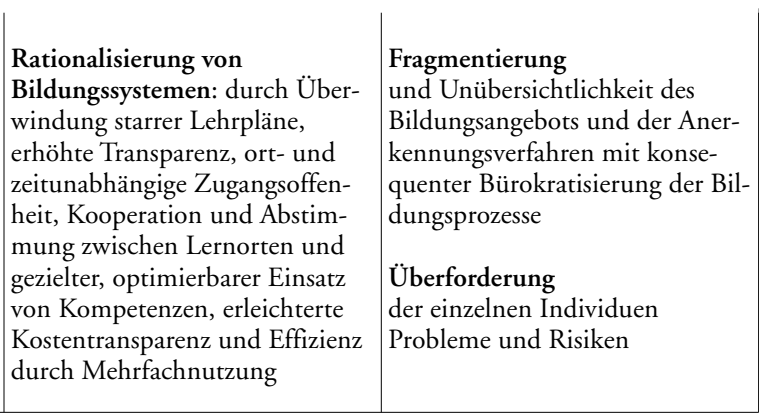

5. Unter den stichwortartig aufgeführten Chancen und Risiken der Modularisierung lassen sich einige aus der Perspektive der curricularen Ausgestaltung von Lehr- und Lernprozessen näher betrachten und einsichtig machen. Hiezu gehören insbesondere jene, die mit der Auswahl und der Anordnung von Bildungsinhalten sowie der Prüfung und Anerkennung von Bildungsleistungen zu tun haben. Wir vergleichen dabei den traditionellen mit dem modularen Ansatz, so wie sie in Abb. 1 und 2 sehr skizzenhaft dargestellt werden. Es gilt zu beachten, dass der hier als traditionell bezeichnete Ansatz v.a. für Allgemeinbildung und die obligatorische Schule typisch ist, während sich, wie bereits gesehen, die berufsorientierten Bereiche des Bildungssystems in den letzten Jahren entschieden in Richtung modularer Ansatz bewegen.

Im traditionellen Ansatz setzen die vier Etappen des curricularen Systems bei der Auswahl der Bildungsinhalte (Fenster 1) an. Entscheidend ist, dass deren Definition von den bestehenden Wissenssystemen, d.h. von den Disziplinen der Wissenschaften und generell vom tradierten Wissen ausgeht. Es kommt also jene grundlegende Logik ins Spiel, die dem tradierten Wissen zu Grunde liegt, so wie es sich historisch im System der Wissenschaften konstituiert hat. Die sozialen Akteure (Fenster 4), bzw. die Anforderungen der Praxis, tragen, wenn überhaupt nur indirekt und mit generischen Bezügen zur Auswahl und Definition der Bildungsinhalte bei. Mit der curricularen Ausgestaltung (Fenster 2) werden die wissenschaftlichen Disziplinen zwar nicht einfach 1 zu 1 in Unterrichtsfächer umgesetzt und es findet eine didaktische Vermittlung statt, letzten Endes wird aber der Lehrplan von deren epistemologischen Identität geprägt. Die angebotenen Bildungsinhalte erfahren so eine doppelte Legitimation: Zum einen rechtfertigen sie sich aufgrund ihres Status im wissenschaftlichen Kontext, zum anderen entspringt ihre Sinnhaftigkeit aus der wissenschaftlichen Struktur selbst, d.h. sie werden von Innen her legitimiert. So gehört z.B. ein bestimmtes mathematisches Wissen deshalb zum Lehrplan, weil es notwendiger Bestandteil der mathematischen Wissenssystematik ist. Der Wert des Wissens und seine bildende Funktion sind, pointiert ausgedrückt, der Wissensstruktur immanent. Dies ist also die Logik der traditionellen Lehrpläne, die das Bildungssystem (Fenster 3) mit ihrer 
Fachorientierung prägen und der abstrakten Lern- und Buchschule ihren Stempel aufdrücken 25 .

Auf dieser Basis entsteht ein Angebot, das von den sozialen Akteuren (Fenster 4) abgeholt wird und die Erlangung von bestimmten Zertifizierungen und von spezifischen bzw. Gesamtqualifikationen ermöglicht. Damit wird eine angebotsorientierte Grundstruktur des (v.a.) allgemein bildenden Kanons geprägt. Als entscheidend erweist sich dabei das kulturelle Erbe an sich, das, weitgehend unabhängig von spezifischen gesellschaftlichen, insbesondere von der Arbeitswelt und vom Beschäftigungssystem bestimmten Bedürfnissen, weiter tradiert werden und zur Bildung von allgemein mündigen Individuen und Bürgern führen soll. Lehr- und Lernprozesse zielen nicht direkt auf die Entwicklung von bestimmten gesellschaftlich erwünschten Kompetenzen ab. Die immanente Rechtfertigung der Bildungsinhalte prägt so die Input-Orientierung des Systems mit.

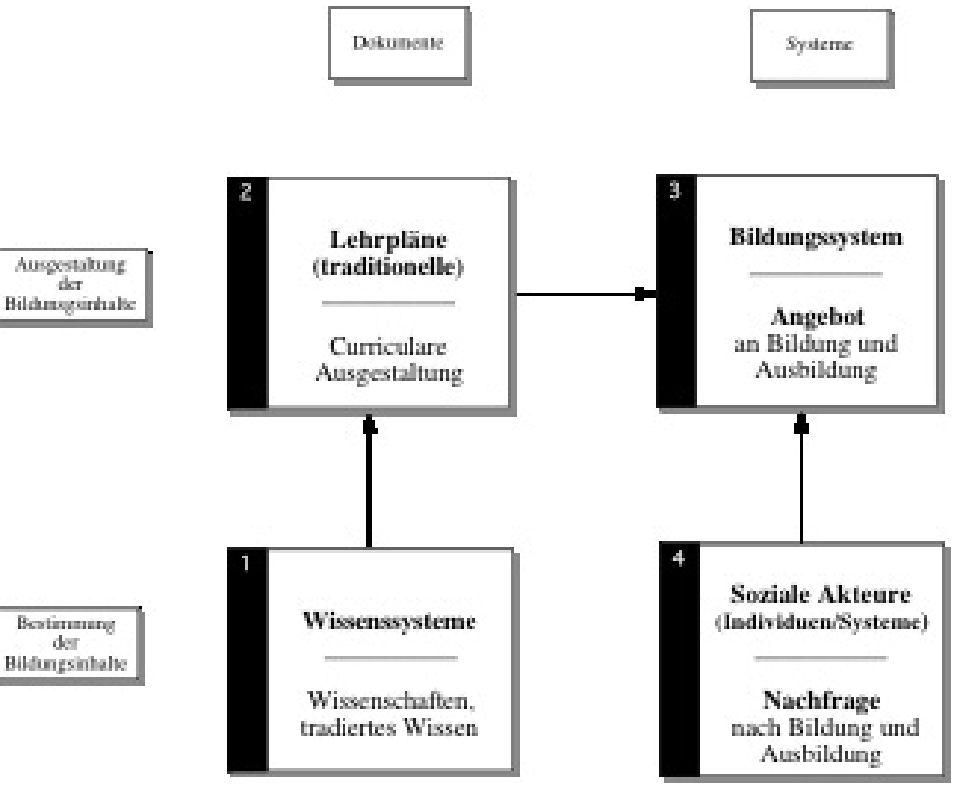

Abb.1: Curriculumsystem: Traditioneller Ansatz - va. Allgemeinbildung

Der modulare Ansatz bildet das organisatorisch-strukturelle Gerüst für ein curriculares System, welches bei den sozialen Akteuren (Fenster 1) ansetzt. Die Bestimmung von womöglich zukunftsorientierten Bedürfnissen aus dem sozialen, kulturellen und beruflichen Alltag stellt die Voraussetzung dar, für die Definition der Bildungsinhalte in Form von Kompetenzen. Was die Berufsbildung anbelangt, so wird in der Regel von einer Funktionsanalyse des Handlungsfeldes ausgegangen, die zur Identifizierung von Kenntnissen, Fähigkeiten und Haltungen führt, welche zur Bewältigung von beruflich relevanten Situationen befähi- 
gen sollen (vgl. AAVV, 1999, p. 7 u. 52). Eine solche funktionale Bestimmung scheint auch immer häufiger als Voraussetzung für die obligatorische Grundund Allgemeinbildung in Frage zu kommen. So lässt sich dies bei der zunehmenden Kompetenzorientierung der Grundschule beobachten, die u.a. durch den Einfluss von internationalen Monitoringssystemen wie PISA begünstigt wird ${ }^{26}$. Damit wird in der Auswahl der Bildungsinhalte eine neue Logik eingeführt, die von der Praxis, d.h. letzten Endes von deren Verwendungskontext her determiniert wird. So rechtfertigen sich Bildungsinhalte nicht mehr primär aufgrund ihres wissenschaftlichen Ursprungs, sondern aus dem Beitrag, den sie zur Entwicklung von praxisorientierten, zur Bewältigung von Lebenssituationen notwendigen Kompetenzen leisten. Dem Kanon soll eine neue, kompetenzorientierte Epistemologie zu Grunde liegen, die sich entscheidend auf die curriculare Ausgestaltung auswirken kann: Die traditionelle, starre Fächerstruktur kann aufgebrochen werden und etwa auf der Basis des Modulansatzes themenoder kompetenzorientiert neu konzipiert werden. Demzufolge finden die sozialen Akteure im Bildungssystem (Fenster 4) Angebote, welche ihren Bedürfnissen und Anforderungen entsprechen.

Von der Lernkonzeption her, wird ein solcher Ansatz die Handlungsorientierung (vgl. Anm. 25), vom System her die Output-Orientierung privilegieren.
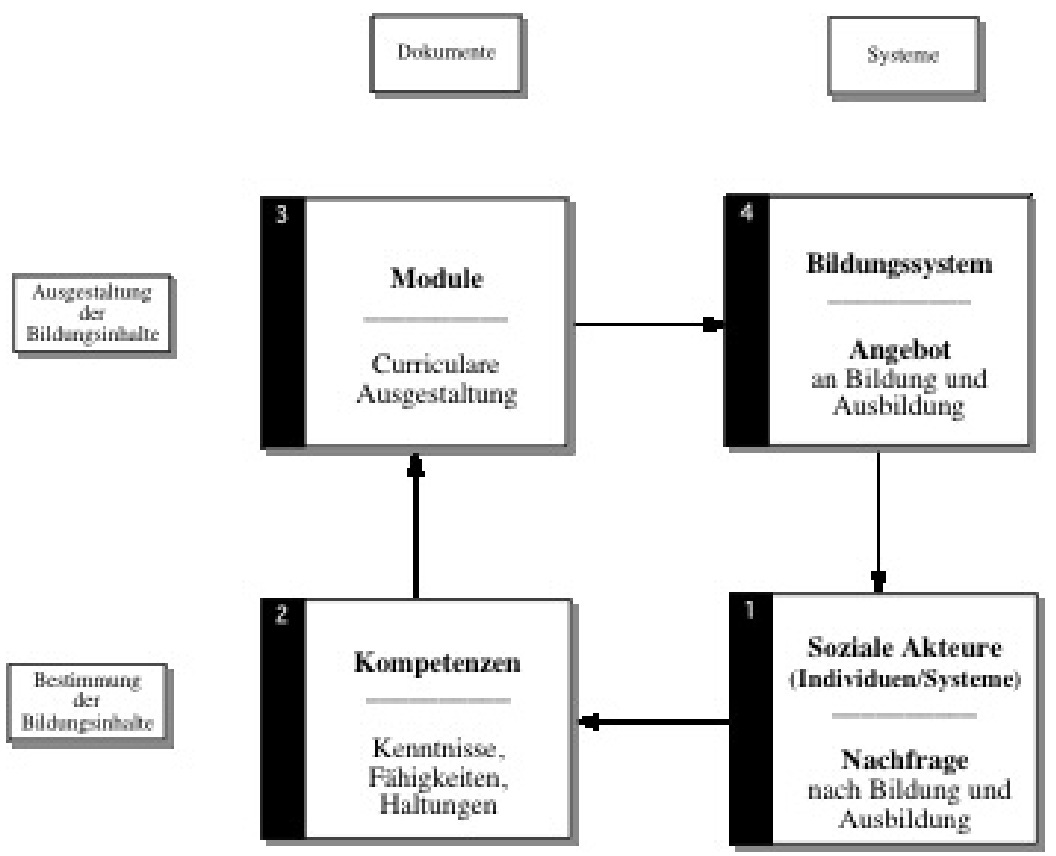

Abb. 2: Curriculumsystem: Modularer Ansatz - v.a. Berufsbildung 
Die zwei dargestellten idealtypischen Ansätze der Curriculumkonstruktion entsprechen so kaum der Komplexität in der Wirklichkeit, setzt man sie aber auf den Poolen eines Kontinuums an, so lassen sie die Möglichkeit von unzähligen Mischformen offen. Ihre Anschaulichkeit erlaubt es nun, einige Fragestellungen auf den Begriff zu bringen.

- Zur Frage der Funktionalisierung und Kompetenzorientierung. Werden die Bildungsinhalte nachfrageorientiert, d.h. ausgehend von den Bedürfnissen der jeweiligen gesellschaftlichen Praxis (Beruf, Alltag) bestimmt, so stellen sich Probleme mindestens hinsichtlich einer doppelten Einengung: Zum einen beziehen sie sich auf bestimmte, historisch vorgegebene Verhältnisse und können somit einen höchst konservativen Charakter annehmen. Zum anderen, und v.a. in der beruflichen Bildung, werden sie zweckorientiert den Bedingungen des Arbeitsplatzes und den kurzfristigen Interessen des Arbeitsprozesses untergeordnet. Diese doppelte Reduktion kann von einem eng gefassten Begriff der Kompetenzen, der ausschliesslich auf Fähigkeiten oder Fertigkeiten (Englisch: skills) ausgerichtet ist, stark begünstigt werden. So ist es im englischen NVQ-Modell geschehen, wo die «berufliche Bildung auf die Rolle der Arbeitskraft am Arbeitsplatz reduziert (wurde), beurteilt anhand von Standards of Competence, die von Industry Lead Bodies (ILBs) geschaffen wurden. (...) Das britische Konzept von Competence führt zu einer Reduzierung von beruflicher Bildung, lediglich den Anforderungen des Arbeitsplatzes zu entsprechen» (Bünning, Hortsch et al., 2000, p. 71; vgl auch Jessup, 1991, 15 zit. in Ertl \& Sloane, 2003, p. 95 )

Dadurch schlägt das klassische Theorie-Praxis Problem der Bildung ins Gegenteil um: Schmalspurigkeit entsteht nicht mehr durch Abstraktion und mangelnden Realitätsbezug, sondern durch einseitige, zweckorientierte Ausrichtung auf konkrete Anforderungen bzw. berufliche Handlungskompetenz.

- Zur Frage der Fragmentierung bzw. der Aufsplitterung. Werden Bildungsinhalte aufgrund von Bedürfnisanalysen ausgewählt und modular angelegt, so können ihr ursprünglicher epistemologischer Zusammenhang und damit auch ihre immanente Sinnhaftigkeit nicht zum Tragen kommen. Eine neue Legitimation muss geleistet werden, was mindestens auf dreierlei Weise denkbar ist: vom angestrebten Berufsprofil oder Berufskonzept her, sachlogisch vom Verwendungskontext her, oder sie muss schliesslich vom einzelnen, die Bildungsleistungen beanspruchenden Individuum erbracht werden. Etliche Risiken werden dabei manifest: Im Lehr- und Lernprozess ist es häufig sehr schwierig einen sinngebenden Zusammenhang herzustellen, sei es, weil der Verwendungskontext oder das Berufsprofil nur abstrakt vorgegeben ist, sei es, weil einzelne Module von unterschiedlichen Personen mit stark divergierenden Betrachtungsweisen vermittelt werden. Der Mangel an inhaltlicher und zeitlicher Kontinuität im Lehr- und Lernprozess kann die Individuen sehr rasch überfordern, weil sie die Zusammenhänge kaum herstellen können und so nicht nur der Gefahr der Fru- 
stration ausgesetzt werden, sondern auch in die Falle des Arbeitsmarkt-untauglichen, beliebigen Qualfikationssammelsuriums geraten können. Fragmentierung, v.a. wenn sie sehr differenziert vorgenommen wird, etwa in Form von einzelnen Einheiten (units) wie im englischen NVQ-System (Bünning, Hortsch et al., 2000, p. 8), kann nicht nur ins Unermessliche geraten und so einen unerwünschten Bürokratisierungseffekt auslösen, sondern auch in didaktischer Hinsicht sehr problematisch sein. Mangelhaft strukturiertes und kohärentes Wissen jenseits des fachspezifischen Zusammenhanges erschwert die Lern- und Behaltensprozesse und kann sich leicht negativ auf die Motivation aller an Lehr- und Lernprozessen beteiligten Akteure und auf die Effizienz des Lernens auswirken. Die Erfahrungen zeigen überdies, wie modulare Fragmentierung die traditionellen Ausbildnerrollen stark beeinträchtigen: Einerseits bedingt sie die Neudefinition solcher Rollen, indem etwa Modulanbieter äusserst fachspezifisch auftreten, andererseits löst sie das Entstehen neuer Rollen aus (Lernbegleiter, Mentor, Tutor, Coacher), die den Lernenden helfen sollen, sowohl Sinnzusammenhänge herzustellen, als auch unerlässliche persönliche, methodologische und metakognitive Kompetenzen zu entwickeln (z.B. die Fähigkeit selbständig und selbstverantwortlich auf disziplinierter und kontinuierlicher Weise zu arbeiten). Mögliche Strategien zur Sicherung von Sinngebung, die Neudefinition von herkömmlichen Ausbildnerrollen sowie die Schaffung von neuen Rollen werden von Luca Bausch in seinem Artikel in diesem Heft, ausgehend von zahlreichen konkreten Erfahrungen in modularen Bildungsgängen, diskutiert. Im Beitrag von Cantoni und Botturi werden überdies die e-learning Ressourcen als Mittel zur Abfederung zahlreicher Probleme der Moduldidaktik, vor allem im kommunikativen Bereich, vorgestellt.

- Zur Frage der Normierung und Homogenisierung. Modulare Bildungsangebote rechtfertigen sich u.a. wenn sie eine breite, auch international wirksame Anerkennung der Bildungsleistungen ermöglichen. Dies kann dazu führen, dass Bildungsinhalte nicht nur an bestimmte quantitative Standards gebunden werden, so z.B. mit den Credits des ECTS, sondern auch inhaltliche Angleichungen erfordern. Paradoxerweise könnte sich dadurch ein gepriesener Vorteil vom modularen Ansatz ins Gegenteil kehren: Anstatt Vielfalt und Individualisierung zu ermöglichen, bewirkt er, in inhaltlicher Hinsicht, die Voraussetzungen für eine kulturelle Normierung und Homogenisierung. Um es bildhaft auszudrücken: Normmöbel können durchaus einen hohen qualitativen und ästhetischen Standard aufweisen, ihnen haftet aber unauslöschlich der Makel der Massenproduktion an.

Werden modulare Bildungsangebote an Standards gebunden, die für die Transparenz und Anerkennung unabdingbar sind, dann verursacht deren praktische Umsetzung auf breiter nationaler und internationaler Skala einen nicht zu unterschätzenden bürokratischen Aufwand, der sich auf die Zusammenarbeit zwischen Bildungsinstitutionen und -partnern hemmend auswirken kann. 
6. Modularisierte Bildungsgänge und -systeme sollen ihrem Anspruch nach helfen, gesellschaftlicher wie biographischer Vielfalt gerecht zu werden, indem sie jene strukturellen Anpassungen favorisieren, welche, auch durch die neuen Informationstechnologien begünstigt, einen offenen, flexiblen, d.h. ort- und zeit-unabhängigen Zugang zur Bildung möglich machen. Insofern kann Modularisierung als authentischer Ausdruck der Moderne betrachtet werden, ja der Postmoderne soweit sie eine radikale Individualisierung und Flexibilisierung der Lebensgestaltung präkonisiert. Gleichsam bietet sie sich in verschiedenen Varianten an als Strategie zur Rationalisierung und Effizienzsteigerung von Bildungsprozessen prinzipiell auf allen Stufen und Ebenen der Bildung, national wie supranational. So kann sie etwa als wichtige Voraussetzung zur Schaffung eines europäischen Bildungsraumes, v.a. im Hochschul- und im berufsbildenden Bereich betrachtet werden, da modulare Systeme die zu einer internationalen Anerkennung von Qualifikationen notwendigen Standardisierungsverfahren begünstigen. Und dennoch wird «Modularisierung nirgends einfach als Positivum begrüsst» (Gonon, 2002, p. 371) und darf es wohl auch kaum, denn die mit ihr verbundenen Probleme und Risiken sind zu manifest, dass man ihre ambivalente Natur übersehen könnte. Solche Probleme siedeln sich auf zwei in sich verschränkten Ebenen an, nämlich der bildungspolitischen (oder genereller ordnungspolitischen) Ebene der strukturellen Organisation von Bildungssystemen und der Ebene der curricularen und didaktischen Gestaltung von Bildungsprozessen. Mit der Modularisierung geht offensichtlich eine verstärkte Nachfrageorientierung des Bildungsangebots (und Output-Orientierung des Systems) einher: Unbeschadet der Vorteile, die eine solche Orientierung, v.a. im Berufsbildungsbereich mit sich bringt, darf das Risiko einer einseitigen Unterordnung von Bildung unter die kurzfristigen, sektoriellen und funktional bestimmten Interessen des Marktes bzw. des Arbeitsplatzes nicht unterschätzt werden ${ }^{27}$. Auch die Anerkennung und Verwertbarkeit der Modulzertifikate auf dem Bildungsund Arbeitsmarkt können das System an die Grenzen der Belastbarkeit führen. Auf der curricularen und didaktischen Ebene kommt das Problem der Auswahl und Bestimmung der Bildungsinhalte nochmals prägnant zum Ausdruck, da sowohl die Kompetenzorientierung als auch die Fragmentierung des Wissens das Risiko des Verlusts von Kohärenz, Kontinuität und v.a. Sinngebung in sich bergen. Auffällig problematisch können schliesslich die Auswirkungen auf die Gestaltung der Lehr- und Lernprozesse sein, vorab auf die inhaltliche und didaktische Abstimmung und Vernetzung zwischen den Modulen und auf die kommunikativen Prozesse.

Wie Matthias Pilz in seinem Beitrag in diesem Heft aufzeigt, sind solche Probleme v.a. mit radikalen Modulkonzepten verbunden. Die Erfahrung der letzten Jahre, etwa in Schottland, wo die Berufsbildung nach einem solchen Konzept reformiert wurde, zeigt aber, dass extreme Modularisierung keinen Bestand hat und dass vielmehr nach praktikablen und flexiblen Mischformen gesucht wird.

Dazu sind aber noch wenig Erkenntnisse vorhanden. Es ist kein Zufall, dass 
in dieser Einführung, aber auch in den Beiträgen dieses Heftes nur sporadisch auf empirische Befunde zurückgegriffen wird. In der Tat haben die zahlreichen Implementationsprojekte der letzten Jahrzehnte wohl zu vielen Vergleichsanalysen geführt, hingegen sind uns nur vereinzelte gezielte oder umfassende empirische Untersuchungen zu den Auswirkungen von modularen Bildungsangeboten bekannt ${ }^{28}$. So überrascht es auch kaum, wenn z.B. in zwei der neuesten deutschsprachigen Handbücher zur Bildungsforschung (Tippelt, 2002; Helsper \& Böhme, 2004), die Problematik keine Erwähnung findet und die Begriffe «Modul» bzw. «Modularisierung» oder auch "Baukastensystem» in deren Sachregister nicht auftaucht. Es ist deshalb ein dringendes Desideratum, dass die komparative Forschung zu modularen Systemen mit quantitativen und qualitativen Untersuchungen eigentlich zum ganzen Spektrum der in dieser Einführung thematisierten Fragen ergänzt werden sollte.

\section{Anmerkungen}

1 Erstmals scheinen Module aber bereits 1869 an der Harvard University angeboten worden zu sein (vgl. Badley \& Marshall, 1995, p. 17)

2 Ausführliche Informationen dazu: http://europa.eu.int/comm/education/programmes/socrates/ects_en.html

3 Ausführliche Informationen dazu: http://europa.eu.int/comm/education/copenhagen/index_de.html

4 Die modularen Erfahrungen im Berufsbildungssystem in der Schweiz wurden in einem dokumentarisch sehr wertvollen Beitrag von Emil Wettstein aufgearbeitet. Dieser Beitrag ist auf der Homepage der SZBW verfügbar

http://www.szbw.ch/Revues/J05_2/index.html Vgl. auch AAVV, 1999, p. $16 f$

5 Unterdessen war das BIGA aufgelöst und vom BBT mit durchaus ähnlichen Funktionen ersetzt worden.

6 In Wirtschaftskreisen wurden auch Bedenken zur effektiven Akzeptanz einer Modulzentrale laut (Dubs 1999, p. 10)

7 Dubs als Gutachter des Schlussberichts aus der Pilotphase hinterfragt zuerst die Vision, die dem vorgeschlagenen Modell zugrunde liegt: «...beinhaltet die Studie eine Vision, von der trotz dem guten Bericht nicht gesagt werden kann, ob sie langfristig tragfähig ist. Je nachdem, welchen Stellenwert man dieser Vision gibt, wird man zu anderen Schlussfolgerungen gelangen.» Ferner äussert er sich auch kritisch zur Idee institutionell die Modulzentrale als Verbundaufgabe anzugehen: "Man könnte die Frage aufwerfen, ob es nicht sinnvoller wäre, dem BBT grössere Kompetenzen (z.B. Qualitätssicherung der Module, Modulprüfung) zu übertragen, ohne eine komplizierte halbstaatlich/halbprivate Organisation aufzubauen, die letztlich mit Sicherheit viele Kompromisslösungen vollziehen muss, welche dem angestrebten Ziel zuwiderlaufen.» (Dubs, 1999, p. 3-4) Für die kritische Auseinandersetzung mit dem vorgeschlagenen Konzept vgl. man auch die Expertise von Dieter Euler (Euler, 1999).

8 Der Verein «modula» wurde aufgelöst. Unterdessen hat sich eine «Clearingsstelle moduQua» gebildet, die grösstenteils von privaten Vereinen und Organisationen getragen wird und versucht, das modulare Angebot in der beruflichen Weiterbildung nach einheitlichen Grundsätzen zu koordinieren und die Akkreditierung zu regeln. Vgl. www.moduqua.ch

9 www.i-ch.ch 
10 Z.B. bei den Pharmaassistentinnen, die die Programme ihrer Grundbildung auf der Basis eines Kompetenz- und Ressourcenorientierten Ansatzes revidieren und das Curriculum teilweise modular aufbauen (vgl. Ghisla, Bausch et al., 2005; Bausch, Boldrini et al., 2005)

$11 \mathrm{Zu}$ erwähnen ist etwa das Projekt zur modularen Neugestaltung des Lehrplanes der Primarschule im Kanton Genf (Wandfluh \& Perrenoud, 1999).

$12 \mathrm{Im}$ Deutschen werden teilweise noch die in den 70er Jahren eingeführten Termini «Baukasten», «Bauelemente» und «Baukastensystem» in der Regel als Synonyme zur Modulterminologie verwendet. Zur Definitionsfrage vgl. auch: Gonon 2002, 367 ff; Kloas 1997, 2 ff; AAVV 1999, 9 u.17, Balducci 2003, 4 f .

13 Balducci, der den Stand der italienischen Diskussion zur modularen Didaktik analysiert, hebt vier Eigenschaften von didaktischen Modulen hervor: segmentalità (Segmentierung), d.h. ein Modul ist Teil (Segment) einer Ganzheit; specificità (Eigenart), d.h. ein Modul zielt auf präzise, kompetenzorientierte Lernziele; componibilità (Zusammensetzbarkeit), d.h. ein Modul kann mit unterschiedlichen Modulen so kombiniert werden, dass verschiedene Lernparcours entstehen können; capitalizzabilità (Akkumulierbarkeit), d.h. ein Modul kann in verschiedenen Bildungskontexten verwertet werden. (Balducci, 2003, p. 5)

14 Die Credits des ECTS betragen 25-30 Stunden Arbeitsaufwand. Ein Credit entspricht aber nicht automatisch einem Modul.

15 Es bestehen dabei etwa drei Möglichkeiten: a) ein Modul gilt aufgrund von bestimmten Kriterien einfach als bestanden oder nicht bestanden, b) die Beurteilung erfolgt aufgrund einer Skala mit verschiedenen Niveaus, c) das gleiche Modul wird auf verschiedenen $\mathrm{Ni}$ veaus neu definiert.

16 Vgl. auch Gonon, der eine anders strukturierte Typologie verwendet (Gonon, 2002, p. 372)

17 M. Pilz in seinem Beitrag verwendet den Begriff Berufskonzept, da damit das Beibehalten eines Berufsprofils als Ausgangspunkt einer Modularisierung deutlicher wird.

18 Für weitere vergleichende Untersuchungen vgl.: Die Dokumentation der CEDEFOP (European Centre for the Development of Vocational Training / Centre Européen pour le Développement de la Formation Professionnelle - www.cedefop.eu.int -, Bünning (2000) für eine ausführliche Darstellung des britischen Modells und die Literaturangaben in Ertl \& Sloane (2003).

19 Die Verfasser des Schlussberichts zur beruflichen Weiterbildung im Baukastensystem in der Schweiz drücken sich wie folgt aus: «Die Modularisierung in der Grundbildung wird im Verlauf der nächsten Jahre Realität werden. Sie wird sich von «den Rändern her» entwickeln und sich von dort her ausbreiten. (AAVV 1999, p. 76)

20 Es darf nicht vergessen werden, dass der Terminus Modul aus dem Lateinischen stammt und soviel wie Mass und Masseinheit bedeutet. Damit muss ein Modul von seinen Eigenschaften her einheitlich und invariabel sein, nur so kann es im Voraus berechenbar sein.

21 Gonon bezeichnet die Modularisierung als «...das Resultat eines Individualisierungsschubes, eines Wandels der Arbeitswelt und eine (nicht-intendierte) Folge der Reflexivität des Bildungswesens.» (Gonon, 2002, p. 374)

22 Für eine Diskussion der Beziehung zwischen Modularisierung und Modernisierung sei auf Gonon (2002, p. 375 ff) verwiesen. Die für diese Diskussion notwendige sozialkritische Zeitdiagnose der Moderne und Postmoderne liefern u.a. die Arbeiten von Ulrich Beck (1986), Richard Sennet (1998), Axel Honneth (1994) und Zygmut Bauman (1999).

$23 \mathrm{Zu}$ beachten ist aber auch, dass gerade solche Tendenzen widersprüchliche und ambivalente Züge aufweisen, zumal die Globalisierung über die Wirkung des Marktes und des Konsums gleichzeitig zur Homogeneisierung von Lebensgewohnheiten führt.

24 Für die differenzierte Darstellung von Vorteilen und Risiken von modularen Ansätzen vgl. man: Euler, 1999, p. 29-30; Ertl \& Sloane, 2003, p. 96; Kloas, 1997, p. 25 ff.

25 Selbstverständlich wurde diese Logik im pädagogischen Diskurs und in der Praxis der Lehrplankonstruktion und des Unterrichts vielfach in Frage gestellt. Ebenso zahlreich sind 
die Alternativen und v.a. Korrektive, die die Pädagogik und Didaktik entwickelt haben, angefangen bei der «Arbeitsschule» über die reformpädagogischen Ansätze zu den sogenannten offenen bzw. handlungsorientierten Curricula mit themenorientiertem Unterricht, mit fächerübergreifenden Modellen oder, auf der didaktischen Ebene, mit dem Projektlernen, dem situierten Lernen, usw.

26 Es sei aber darauf hingewiesen, dass selbst das traditionelle Gymnasium, als Inbegriff einer offenen und nichtfunktionalen Bildung, bereits seit einigen Jahrzehnten Elemente der Bedarfsorientierung aufgenommen hat, so für die Schweiz z.B. bereits mit der Einführung in den 70er Jahren von Maturitätstypen, die an die spätere Berufstätigkeit orientiert sind.

27 Perrenoud geht in kritischer Absicht gar weiter: «So könnte es sein, dass die Modularisierung, die als eine der wichtigsten Innovationen und als didaktisches Hauptkennzeichen der modernen Erwachsenenbildung und der höheren Studien gedacht war, sich zum System entwickelte, das es vehement zu bekämpfen gälte. Das wäre dann der Fall, wenn diejenigen, die es propagieren, es zum Instrument der Rationalisierung und der Deregulierung des Bildungswesens werden liessen.» (Perrenoud, 2002, p. 214).

$28 \mathrm{Zu}$ erwähnen ist die Evaluation der Modularisierung in der Grundbildung Informatik in der Schweiz, die aufschlussreiche Daten etwa zur Akzeptanz und Machbarkeit, zur Ausbildungsqualität und zu den Problemen des Kompetenznachweises liefert (Ochsenbein, 2004).

\section{Bibliographie}

AAVV (1999). Berufliche Weiterbildung im Baukastensystem. Schlussbericht der Pilotphase. Bern: Bundesamt für Berufsbildung und Technologie (BBT).

Badley, G. \& Marshall, S. (1995). 53 Questions and Answers about Modules and Semesters. Bristol: Cromwell Press Ltd.

Balducci, M. (2003). La didattica per moduli. Roma-Bari: Laterza.

Bauman, Z. (1999). La società dell'incertezza. Bologna: il Mulino.

Bausch, L., Boldrini, E. et al. (2005). BIVO Pharmassistentinnen. Bildungsplan und Qualifikationsverfahren. Lugano: SIBP/ISPFP.

Beck, U. (1986). Risikogesellschaft - auf dem Weg in eine andere Moderne. Frankfurt a. M.: Suhrkamp.

Bieri, U., Longchamp, C. et al. (2004). Berufsbildung - in mehrfacher Hinsicht wettberwebfähig. Studie "Schweizer Berufsbildungsdelphi 2004». Bern: gfs.bern.

Bünning, F., Hortsch, H. et al. (2000). Das britische Modell der National Vocational Qualifications (NVQs) . Hamburg: Verlag Dr. Kovac.

Deissinger, T. (1996). Modularisierung der Berufsbildung. Eine didaktisch-curriculare Alternative zum 'Berufsprinzip'. In: Beck, K.\&Achtenhagen, F. Berufserziehung im Umbruch. Didaktische Herausforderungen und Ansätze zu ihrer Bewältigung. Weinheim: Belz 189207.

Dubs, R. (1998). Modularisierung nicht prioritär! Panorama 2(13-14).

Dubs, R. (1999). Berufliche Weiterbildung im Baukastensystem. Gutachten. St. Gallen: Universität St. Gallen.

Ertl, H. \& Sloane, P. F. E. (2003). Beruflichkeit und Modularisierung - Ansätze für die Modernisierung der deutschen Berufsbildung aus dem Vergleich internationaler Modulkonzepte. In: Arnold, R. Berufsbildung ohne Beruf. Hohengehren: Schneider Verlag, 89-128.

Euler, D. (1999). Berufliche Weiterbildung im Baukastensystem. Evaluationsexpertise für das $B B T$. Nürnberg: Universität Erlangen-Nürnberg.

Ghisla, G., Bausch, L. et al. (2005). KoRe. Kompetenzen-Ressourcen: Ein Verfahren zur Entwicklung von Bildungsplänen in der Berufsbildung. Lugano/Zollikofen, SIBP/ISPFP.

Gonon, P. (2002). Arbeit, Beruf und Bildung. Bern: h.e.p. 
Gretler, A., Haag, D. et al. (1972). Die Schweiz auf dem Weg zur Education permanente. Zürich: Benziger.

Helsper, W. \& Böhme, J. (Eds.), (2004). Handbuch der Schulforschung. Wiesbaden: Verlag für Sozialwissenschaften.

Honneth, A. (1994). Desintegration. Bruchstücke einer soziologischen Zeitdiagnose. Frankfurt am Main: Fischer.

Jessup, G. (1991). Outcomes. NVQs and the Emerging Model of Education and Training. London: The Falmer Press.

Kloas, P.-W. (1997). Modularisierung in der beruflichen Bildung. Berlin und Bonn: Bundesinstitut für Berufsbildung

Ochsenbein, H. (2004). Modularisierung in der Grundbildung Informatik. Bericht über die zweite Befragungsrunde 2003/4. Bern: Informatik Berufsbildung I-CH.

Perrenoud, P. (2002). Verwalten statt gestalten - die den modularisierten Bildungsgängen drohende Gefahr. Beiträge zur Lehrerbildung 2: 203-215.

Pütz, H. (1997). "Modularisierung» - das falsche Thema, ungenügend bearbeitet, zur ungeeigneten Zeit. In: Kloas, P.-W. Modularisierung in der beruflichen Bildung. Berlin und Bonn: Bundesinstitut für Berufsbildung 61-75.

Sennett, R. (1998). Der flexible Mensch. Kultur des neuen Kapitalismus. Berlin: Berlin.

Tippelt, R. (Ed.) (2002). Handbuch Bildungsforschung. Opladen: Leske + Budrich.

Wettstein, E. (2005). Modularisierung im Berufsbildungssystem der Schweiz. Zürich. Auf der Homepage der SZBW verfügbar: http://www.szbw.ch/Revues/J05_2/index.html 